Supporting Information for

\title{
Narrow Absorption NIR Wavelength Organic Nanoparticles Enable Multiplexed Photoacoustic Imaging
}

\author{
Hoang D. Lu, ${ }^{\dagger}$ Brian K. Wilson,,$^{\dagger}$ Andrew Heinmiller, ${ }^{,}$Bill Faenza, ${ }^{\gamma}$ Shahram Hejazi, ${ }^{\S}$ and \\ Robert K. Prud'homme*'t
}

${ }^{\prime}$ Department of Chemical and Biological Engineering, Princeton University, Princeton, New Jersey 08544, United States; ${ }^{*}$ FUJIFILM VisualSonics, Toronto, Ontario, Canada; ${ }^{7}$ Persis Science, Andreas, Pennsylvania 18211, United States; ${ }^{\beta}$ Optimeos Life Sciences LLC, Princeton, New Jersey 08544, United States

*to whom correspondence should be addressed; E-mail: prudhomm@princeton.edu

\section{Supplementary Methods}

\section{Synthesis of folate modified polystyrene-block-polyethylene glycol (PS-b-PEG-fA)}

PS-b-PEG-fA was synthesized through the step-wise modification of $1.6 \mathrm{kDa}$ polystyrene-block-5 $\mathrm{kDa}$ polyethylene glycol terminated with a hydroxy functional group (PS-bPEG-OH, Polymer Source Inc., Dorval, Canada). Triethylamine (TEA), methanesulfonyl chloride, N-(3-Dimethylaminopropyl)-N'-ethylcarbodiimide hydrochloride (EDC), N,Ndiisoproylethylamine (DIPEA), N,N-dimethylformamide (DMF) and 24\% ammonium hydroxide solution were purchased from Sigma-Aldrich (St. Louis, Missouri). Methanesulfonyl chloride was stored at $4{ }^{\circ} \mathrm{C}$ and used as received. EDC was stored at $-80^{\circ} \mathrm{C}$ and used as received. TEA was distilled under argon prior to use. Poly(styrene)-block-poly(ethylene glycol)-OH was first converted to PS-b-PEG terminated with an amine functional group (PS-b-PEG-NH ${ }_{2}$ ) via a mesylation reaction. Solid PS-b-PEG-OH powder (500 mg, 0.076 mmoles) was dissolved in dry toluene and azeotropically distilled to remove any water in the block copolymer powder. The polymer was dissolved in $20 \mathrm{~mL}$ of dry toluene following the azeotropic distillation in a septumsealed round-bottom flask. Triethylamine ( $23 \mathrm{mg}, 0.23 \mathrm{mmoles})$ was added under argon to the toluene solution and stirred for 15 minutes. Methanesulfonyl chloride (44mg, 0.379 mmoles) was injected dropwise under argon with magnetic stirring. The flask was covered in foil and allowed to react for 24 hours at room temperature. After 24 hours, the toluene solution was filtered twice through a $0.22 \mu \mathrm{m}$ PTFE filter to remove insoluble TEA chloride salts. The toluene was removed by rotary evaporation and the reaction mixture was redissolved in dichloromethane $(1 \mathrm{~mL})$ and precipitated in diethyl ether $(50 \mathrm{~mL})$ at $-80^{\circ} \mathrm{C}$. The precipitated product was redissolved in dichloromethane and precipitated again in diethyl ether and was then dried in vacuo overnight before NMR analysis in deuterated chloroform. The polystyrene aromatic protons from 6.2-7.25 
ppm (77 H, complicated pattern) serve as an internal standard for normalizing the polymer end group peaks. The $\beta$-methylene group of the PEG adjacent to the mesylate, $4.40(2 \mathrm{H}, \mathrm{t})$ and the methane group of the mesylate (MS), $3.11(3 \mathrm{H}, \mathrm{s})$, show quantitative conversion of all PS-bPEG-OH to PS-b-PEG-MS. PS-b-PEG-MS (400 mg, 0.061 mmoles) was further converted to PS-b-PEG- $\mathrm{NH}_{2}$ by reaction in magnetically stirred $24 \%$ wt ammonium hydroxide aqueous solution $(22 \mathrm{~g}, 24.3 \mathrm{~mL})$ for 96 hours. After 96 hours, the ammonia was evaporated under nitrogen for 72 hours. The $\mathrm{pH}$ of the aqueous reaction mixture was adjusted to 14 with sodium hydroxide and sodium chloride was added to produce saturated brine. PS-b-PEG-NH $\mathrm{N}_{2}$ was extracted with 6 washes of $25 \mathrm{~mL}$ dichloromethane. The dichloromethane washes were combined and concentrated down to $1 \mathrm{~mL}$ before precipitation in $50 \mathrm{~mL}$ of diethyl ether at $80^{\circ} \mathrm{C}$. The product was dried in vacuo overnight prior to NMR analysis in deuterated chloroform. The poly(styrene) aromatic protons from 6.2-7.25 ppm again serve as an internal standard for normalizing the polymer end group peaks. The $\alpha$-methylene group of the PEG adjacent to the amine, $2.87(2 \mathrm{H}, \mathrm{t})$, show quantitative conversion of all PS-b-PEG-MS to PS-b-PEG-NH 2.

PS-b-PEG-fA was synthesized through carbodiimide coupling of folic acid with PS-bPEG-NH 2 . PS-b-PEG-NH2 (150 mg, 0.023 mmoles), folic acid (40 mg, 0.091 mmoles) and EDC (30 mg, 0.15 mmoles) were dried for 12 hours in vacuo before adding N,Ndiisopropylethylamine (DIPEA $15 \mathrm{mg}, 0.12 \mathrm{mmoles}$ ) and $20 \mathrm{~mL}$ of dry N,N-dimethylformamide under argon. The reaction flask was covered in foil and magnetically stirred at $40^{\circ} \mathrm{C}$ for 48 hours. After 48 hours, the DMF was diluted with $40 \mathrm{~mL}$ of deionized water and dialyzed through 6-8 $\mathrm{kDa}$ cutoff dialysis tubing for 4 hours against $8 \mathrm{~L}$ of $5 \mathrm{mM} \mathrm{NaOH}$ and then for 20 hours against $16 \mathrm{~L}$ of deionized water. The dialyzed, micellar solution of polymer was recovered and lyophilized at $-20^{\circ} \mathrm{C}$ and 100 millitorr for approximately 72 hours to dryness. NMR analysis was done in deuterated DMSO, where the poly(styrene) aromatic protons from 6.2-7.25 ppm again serve as an internal standard for normalizing the polymer end group peaks. The two peaks for the ortho-protons in the benzoic acid moiety (conjugated to glutamic acid in whole folic acid), 7.53 $(1 \mathrm{H}, \mathrm{q})$ and $7.63(1 \mathrm{H}, \mathrm{q})$, show quantitative conjugation.

\section{Transmission electron microscopy}

Transmission electron microscopy images were taken with a Philips CM200 TEM equipped with a field emission gun, operated at an accelerating voltage of $200 \mathrm{kV}$. Samples were prepared by drop-casting $5 \mu \mathrm{L}$ of nanoparticle dispersion on a 200 mesh copper TEM grid (Ted Pella, Inc.) and allowing the sample to evaporate overnight. 


\section{In vitro folate targeting}

$\mathrm{KB}$ and $\mathrm{A} 549$ cells were cultured on treated polystyrene dishes at $37^{\circ} \mathrm{C}$ and $5 \% \mathrm{CO}_{2}$ atmosphere in RMPI 1640 media supplemented with 10\% fetal bovine serum. Passaging was accomplished by trypsinization of cells in $0.025 \%$ trypsin and $0.01 \%$ EDTA for 8 minutes at $37^{\circ} \mathrm{C}$ before transfer to fresh media. Confluent A549 and KB cells were dosed with nanoparticles at a concentration of $50 \mu \mathrm{g} / \mathrm{mL}$ in RPMI 1640 culture media supplemented with $10 \% \mathrm{FBS}$ and $1 \%$ penicillin and streptomycin for 3 hours. After 3 hours, unbound particles and media were removed by washing with PBS in triplicate. Cells were stained with a $1 \%$ wt aqueous solution of Eosin Y (Sigma-Aldrich) for 15 minutes before being washed with PBS in triplicate again. Images were acquired on an Axio Observer A1 (Zeiss) inverted epifluorescence microscope equipped with an X-cite 120Q lamp (Excelitas) and Hamamatsu C10600 camera, with camera controller, while images were captured with HCImageLive software (Hamamatsu). The nanoparticle fluorescence signal collected via excitation from 590-650 nm (ET620/60X filter) and emission from 660-740 nm (ET700/75M filter). The Eosin Y cell stain fluorescence signal was collected via excitation from 470-510 nm (ET490/40m filter) and emission from $560 \mathrm{~nm}$ and above (E570LP filter). All filters are from Chroma Technology Group.

\section{Supplementary Figures}

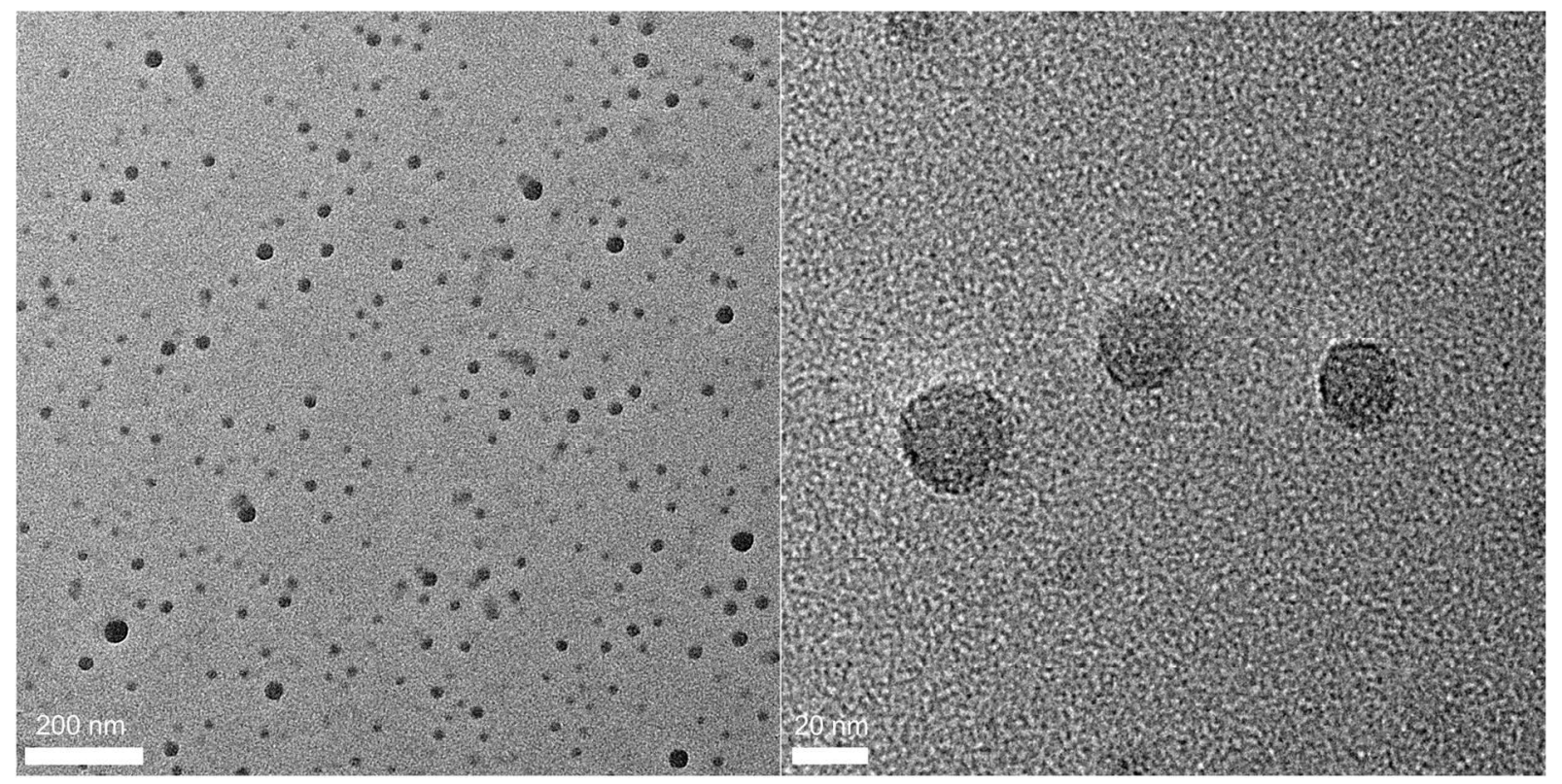

Figure S1. Transmission electron microscopy of formulation one Par788 NPs. Particles have spherical morphologies. 



Figure S2. Optical properties of nanoparticles with core dopants. Normalized absorbancewavelength profiles of (A) Par788, (B) Par830, and (C) Par900 NPs, and zoomed profiles respectively (D-F) when the core contains no additional dopant, polystyrene homopolymer dopant, or vitamin E dopants. The addition of core dopants can slightly influence the absorbance-wavelength spectrum. 


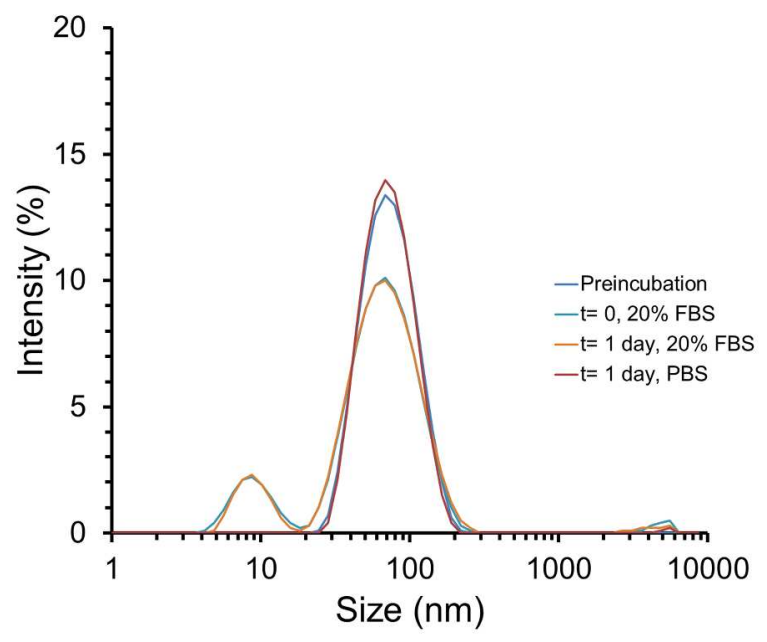

Figure S3. Physical stability of nanoparticles in serum. Dynamic light scattering size distributions of Par788 NPs incubated in $20 \%$ fetal bovine serum (FBS) or PBS at $37^{\circ} \mathrm{C}$ for 24 hours. Small micelle peaks found in samples containing 20\% FBS are from proteins inherently in FBS (Gindy et. al., Biomacromolecules 9, 2705-2711, 2008). The size distribution from Par788 NPs remain the same when incubated in 20\% FBS. Par788 NPs are stable and stay intact in $20 \%$ FBS.

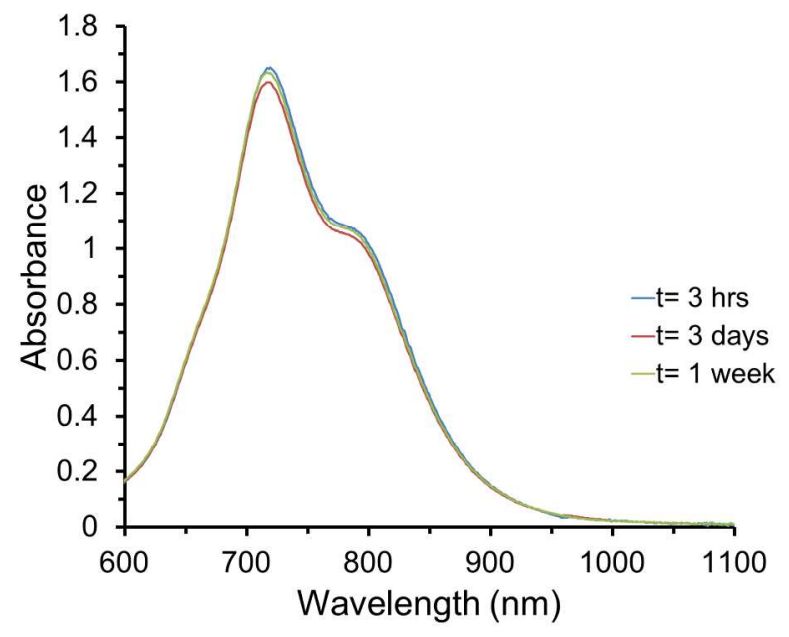

Figure S4. Optical stability of nanoparticles. Absorbance-wavelength profiles of Par788 NPs incubated in PBS at $37^{\circ} \mathrm{C}$ throughout a week. NPs are optically stable and retain the same absorbance profiles during the time course. 


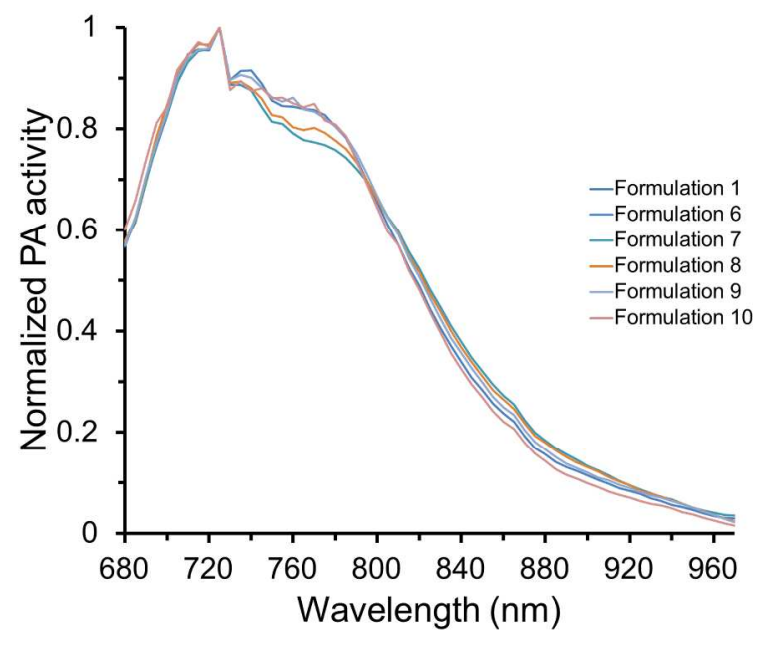

Figure S5. Photoacoustic activity profiles of nanoparticles from different nanoparticle formulations and sizes. Normalized photoacoustic activity-wavelength profiles of Par788 NPs formed from different formulations that result in NPs 40 to $90 \mathrm{~nm}$ in size. The overall profile of the photoacoustic-wavelength spectra remains similar throughout all formulations and sizes tested. The exact formulations and sizes are detailed in Table S1.

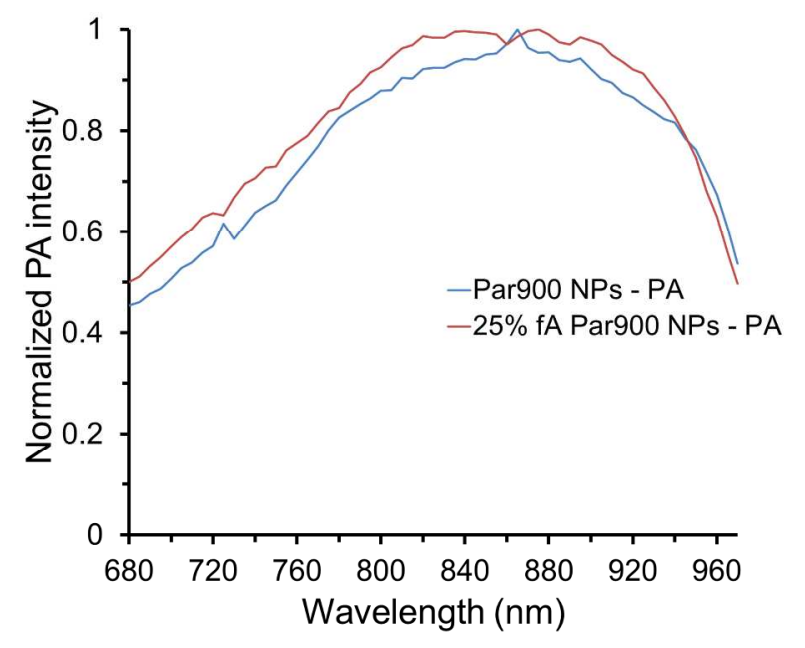

Figure S6. Normalized photoacoustic activity profiles of Par900 NPs. Folate surface functionalized Par900 nanoparticles display comparable photoacoustic activity profiles when compared with non-targeted Par900 nanoparticles. 


\section{Supplementary Tables}

\begin{tabular}{|c|c|c|c|c|c|c|c|c|c|c|}
\hline \multirow{2}{*}{ Formulation } & \multicolumn{2}{|c|}{ Interfacial Stabilizer } & \multicolumn{2}{|c|}{ Photoacoustic Agent } & \multicolumn{2}{|c|}{ Co-core } & \multicolumn{4}{|c|}{ NP Properties } \\
\hline & Block copolymer & Conc $(\mathrm{mg} / \mathrm{mL})$ & Dye & Conc $(\mathrm{mg} / \mathrm{mL})$ & Dopant & Conc $(\mathrm{mg} / \mathrm{mL})$ & Z-Diameter (nm) & PDI & $\varepsilon\left(\mathrm{mL} \mathrm{mg}^{-1} \mathrm{~cm}^{-1}\right)$ & $\alpha\left(\mathrm{Pa} \mathrm{A}^{-1}\right)$ \\
\hline 1 & $\mathrm{PS}_{1.6 \mathrm{k}}-\mathrm{b}-\mathrm{PEG} \mathrm{G}_{5 \mathrm{k}}$ & 3.0 & Par788 & 3.0 & - & & $49 \pm 1.1$ & $0.17 \pm .01$ & 37.9 & 133 \\
\hline 2 & $P S_{1.6 k}-b-P E G_{5 k}$ & 3.0 & Par830 & 3.0 & - & & $39 \pm 0.7$ & $0.14 \pm .03$ & 21.7 & 120 \\
\hline 3 & $\mathrm{PS}_{1.6 \mathrm{k}}-\mathrm{b}-\mathrm{PEG} \mathrm{G}_{5 \mathrm{k}}$ & 3.0 & Par900 & 3.0 & - & & $52 \pm 0.3$ & $0.10 \pm .01$ & 13.7 & 140 \\
\hline 4 & $\mathrm{PS}_{1.6 \mathrm{k}}-\mathrm{b}-\mathrm{PEG} \mathrm{G}_{5 \mathrm{k}}$ & 3.0 & Par788 & 3.0 & $\mathrm{PS}_{1.8 \mathrm{k}}$ & 3.0 & $65 \pm 0.4$ & $0.12 \pm .02$ & 43.2 & 135 \\
\hline 5 & $\mathrm{PS}_{1.6 \mathrm{k}}-\mathrm{b}-\mathrm{PEG} \mathrm{G}_{5 \mathrm{k}}$ & 3.0 & Par788 & 3.0 & VitE & 3.0 & $66 \pm 0.5$ & $0.13 \pm .01$ & 38.6 & 127 \\
\hline 6 & $P S_{1.6 \mathrm{k}}-\mathrm{b}-\mathrm{PEG} \mathrm{G}_{5 \mathrm{k}}$ & 1.5 & Par788 & 3.0 & - & & $62 \pm 1.4$ & $0.15 \pm .02$ & 38.6 & 132 \\
\hline 7 & $\mathrm{PS}_{1.6 \mathrm{k}}-\mathrm{b}-\mathrm{PEG}_{5 \mathrm{k}}$ & 6.0 & Par788 & 3.0 & - & & $42 \pm 0.9$ & $0.21 \pm .01$ & 37.1 & 112 \\
\hline 8 & $\mathrm{PS}_{1.6 \mathrm{k}}-\mathrm{b}-\mathrm{PEG} \mathrm{G}_{5 \mathrm{k}}$ & 6.0 & Par788 & 6.0 & - & & $52 \pm 0.5$ & $0.17 \pm .02$ & 37.0 & 117 \\
\hline 9 & $\mathrm{PS}_{1.6 \mathrm{k}}-\mathrm{b}-\mathrm{PEG} \mathrm{G}_{5 \mathrm{k}}$ & 9.0 & Par788 & 9.0 & - & & $66 \pm 0.8$ & $0.15 \pm .02$ & 33.2 & 141 \\
\hline 10 & $\mathrm{PS}_{1.6 \mathrm{k}}-\mathrm{b}-\mathrm{PEG} \mathrm{G}_{5 \mathrm{k}}$ & 3.0 & Par788 & 9.0 & - & & $88 \pm 1.1$ & $0.28 \pm .08$ & 32.6 & 185 \\
\hline 11 & $\begin{array}{c}25 \% \text { PS }_{1.6 k} \text {-b-PEG } \\
75 \% \text {-fA, } \\
75 \% \text { PS1.6k-b-PEG5k }\end{array}$ & 5.0 & Par900 & 5.0 & - & & $59 \pm 0.3$ & $0.09 \pm .01$ & 14.8 & 145 \\
\hline
\end{tabular}

Table S1. Summary of nanoparticle formulations and nanoparticle properties. The concentrations of stabilizers, photoacoustic reagents, and co-core concentrations in THF organic feed streams used during Flash NanoPrecipitation and the properties of the resultant particles formed are shown. Reported nanoparticle sizes and polydispersities are averages of three measurements, and errors are standard deviations from three measurements. The absorbance extinction coefficient $\varepsilon$ reported is based on the normalizing the peak absorbance values to the mass of Par dye mass concentration within the NPs. The specific photoacoustic activity $\alpha$ is based on normalizing the photoacoustic activity intensity with the absorbance value of the NP. 